\title{
Forearm porphyrin levels evaluated by digital imaging system are increased in patients with systemic sclerosis compared with patients in pre-clinical stage
}

\author{
Kayoko Tabata ${ }^{1}$, Chikako Kaminaka ${ }^{2, *}$, Misaki Yasutake ${ }^{1}$, Ryo Matsumiya ${ }^{1}$, Yutaka Inaba ${ }^{2}$, \\ Yuki Yamamoto ${ }^{2}$, Masatoshi Jinnin², Takao Fujii ${ }^{1}$ \\ ${ }^{1}$ Department of Rheumatology and Clinical Immunology, Graduate School of Medicine, Wakayama Medical University, Wakayama, Japan; \\ ${ }^{2}$ Department of Dermatology, Graduate School of Medicine, Wakayama Medical University, Wakayama, Japan.
}

SUMMARY We hypothesized that changes in skin characteristics on the forearm could be useful for early diagnosis of systemic sclerosis (SSc). We used VISIA digital imaging system to investigate this possibility for the first time. Twenty-eight Japanese patients who were diagnosed with typical or very early diagnosis of SSc (VEDOSS) were enrolled in this study, and ten age- and gender-matched patients with other disorders were included as a control group. Eight skin characteristics were analyzed. Our method of evaluating forearm skin characteristics was shown to be reproducible. The scores of WRINKLES, TEXTURE, PORES, and PORPHYRINS were higher in SSc subjects with sclerotic forearm skin (SSc forearm+; 11.004, 5.116, 3.230, and 0.084, respectively) and those without (SSc forearm-: 11.915, $4.898,2.624,0.0616$, respectively) than in the non-SSc control subjects $(10.075,4.496,2.459,0.0223$, respectively). Also, the scores of SPOTS, TEXTURE, PORES, UV SPOTS, BROWN SPOTS, and PORPHYRINS were elevated in SSc forearm+ (3.182, 5.116, 3.230, 5.761, 6.704, 0.084, respectively) and SSc forearm- patients $(2.391,4.898,2.624,9.835,5.798,0.0616$, respectively) compared with those with VEDOSS $(2.362,4.738,2.234,5.999,4.898,0.0169$, respectively). We found statistical significance in the difference in score of PORPHYRINS between SSc forearm- and VEDOSS groups $(p=0.044)$, and between SSc forearm + and VEDOSS groups $(p=0.012)$. Therefore, they can be used to differentiate VEDOSS from early or mild SSc cases, which is sometimes clinically problematic. Our study also suggests that the porphyrin research will lead to a better understanding of SSc pathogenesis.

Keywords forearm, very early diagnosis of SSc (VEDOSS), porphyria cutanea tarda

\section{Introduction}

Systemic sclerosis (SSc) is characterized by three pathologic features: immunodysfunction/inflammation, vasculopathy, and tissue fibrosis of various organs. Although the exact pathogenesis of SSc remains unknown, tissue fibrosis due to excessive collagen deposition seen in SSc is sometimes irreversible, at least clinically. There is an urgent need to develop new strategies of early diagnosis and careful followup. Early diagnosis is often difficult, however, because of the lack of objective typical skin sclerosis in the early stages. For that purpose, the disease concept of very early diagnosis of systemic sclerosis (VEDOSS) was developed, referring to patients with Raynaud's phenomenon but without skin sclerosis. In addition, for early detection, low serum concentration of carbonic anhydrase 9 (CA9) and microRNA-29 in pre-clinical stage SSc may be utilized as early diagnostic markers $(1,2)$. Also, we found increased sweating levels on finger pads in SSc patients, and demonstrated its clinical significance for early diagnosis of SSc (3).

We hypothesized that changes in skin findings on the forearm could be useful for early diagnosis. Here, we investigate this possibility using a digital imaging system (VISIA) for the first time. VISIA is a facial imaging system by objective computer assessments of eight major skin parameters. As far as we are aware base on search by PubMed using keyword VISIA, systemic sclerosis, and forearm, there has never been any such attempt.

\section{Materials and Methods}

\subsection{Clinical assessment and patient material}

Enrolled in this study were 28 female patients who 
visited Wakayama Medical University between October 2016 and March 2021.

Twenty-one of these 28 patients met the American College of Rheumatology and the European League Against Rheumatism classification criteria (ACR/ EULAR2013) (4), while seven did not fulfill the criteria, but were diagnosed as VEDOSS (5).

Modified Rodnan total skin thickness score (MRSS), a semi-quantitative skin sclerosis assessment tool, was obtained at the time of skin analysis (6). Ten ageand gender-matched patients with other disorders (rheumatoid arthritis: $n=5$, polymyalgia rheumatica: $n=3$, Sjögren syndrome: $n=1$, systemic lupus erythematosus: $n=1$ ) were also included as a control group.

This study was approved by the Wakayama Medical University Institutional Review Board (No.2479), and written informed consent was obtained before patients were entered into this study, in accordance with the Declaration of Helsinki.

\subsection{Photography and forearm skin analysis}

Photographing and forearm skin analysis were performed by objective computer assessments with digital imaging system (VISIA, Canfield Imaging Systems, Fairfield, NJ).

The system consists of imaging chamber with a 15 million pixel resolution camera, which is connected to computer and quantitative analysis software, and has three kinds of light sources: standard incandescent light, ultraviolet (UV) light, and polarized light.

Eight skin characteristics were evaluated: SPOTS, WRINKLES, TEXTURE, PORES, UV SPOTS, BROWN SPOTS, RED AREAS, and PORPHYRINS. A standard flash light is used to identify SPOTS, WRINKLES, TEXTURE, and PORES, whereas an UV flash-light is used to detect UV SPOTS and PORPHYRINS. A cross-polarized flash light is also used to observe BROWN SPOTS and RED AREAS (7).

For example, SPOTS are identified by their color and contrast from the surrounding skin (8). The PORPHYRINS scores reflect fluorescence with UV ray. The definition of other parameters was as described previously (7-12). Average scores were taken from two independent analyses (left and right forearms).

\subsection{Statistical analysis}

Statistical analyses were carried out with KruskalWallis test for analysis of more than three groups, and Mann-Whitney tests were used for the comparison of medians between two groups. Correlations were evaluated by Pearson's correlation coefficient. $P$ values $<0.05$ were considered to be statistically significant.

\section{Results}

\subsection{Clinical features of patients in this study}

Twenty-one female patients with SSc were enrolled in this study. The numbers of SSc patients with or without skin sclerosis of the forearm (SSc forearm+ or SSc forearm-) were $n=6$ and 15, respectively. Seven patients with VEDOSS and ten control patients (rheumatoid arthritis: $n=5$, polymyalgia rheumatica: $n=3$, Sjögren syndrome: $n=1$, systemic lupus erythematosus: $n=1$ ) were also included in this study.

Clinical characteristics of patients included in this study are shown in Table 1. The average age of each group was similar (SSc forearm+: 69.8, SSc forearm-: 68.3, VEDOSS: 65.7, and the control: 68.3). The average ACR/EULAR score was higher in SSc forearm+ group than in the other groups: (SSc forearm+: 20.5, SSc forearm-: 12.5, and VEDOSS: 7.1). Consistently, the average MRSS tended to be increased in SSc forearm+ group (SSc forearm+: 11.3, SSc forearm-: 1.8, and VEDOSS: 0 ). These data thus indicate the credibility of our grouping.

3.2. Comparison of the eight skin characteristics of forearm skin in 4 groups

The eight skin characteristics (SPOTS, WRINKLES, TEXTURE, PORES, UV SPOTS, BROWN SPOTS, RED AREAS, and PORPHYRINS) of forearm skin were analyzed in the four patient groups (SSc forearm+, SSc forearm-, VEDOSS, and the control) by using digital imaging system (VISIA). This is the first report to evaluate forearm skin using VISIA, so we attempted to prove its reproducibility. Each parameter was separately evaluated on the left and right forearms in all subjects, and percentage difference between the two evaluations in each individual was calculated as difference of scores/larger scores $\times 100$ in each patient (Figure 1). The mean percentage differences of each parameter were less than 2-fold, indicating the reproducibility of all parameters by our method, and the mean score of left and right forearms in each patient was evaluated in the following analyses.

The eight parameters were then compared among the four groups. The scores of WRINKLES, TEXTURE, PORES, and PORPHYRINS were higher in SSc forearm+ (WRINKLES; $11.004 \pm 4.287$, TEXTURE; $5.116 \pm 3.254$, PORES; $3.230 \pm 1.591$, PORPHYRINS; $0.084 \pm 0.0891)$ and SSc forearmgroups (WRINKLES; $11.915 \pm 2.747$, TEXTURE; $4.898 \pm 1.864$, PORES; $2.624 \pm 1.299$, PORPHYRINS; $0.0616 \pm 0.108$ ) than in control subjects (WRINKLES; $10.075 \pm 2.370$, TEXTURE; $4.496 \pm 2.373$, PORES; $2.459 \pm 1.590$, PORPHYRINS; $0.0223 \pm 0.0166$ ) (Table 1). Also, the scores of SPOTS, TEXTURE, PORES, UV SPOTS, BROWN SPOTS, and PORPHYRINS were elevated in patients with SSc forearm+ (SPOTS; $3.182 \pm 0.485$, TEXTURE; $5.116 \pm 3.254$, PORES; 


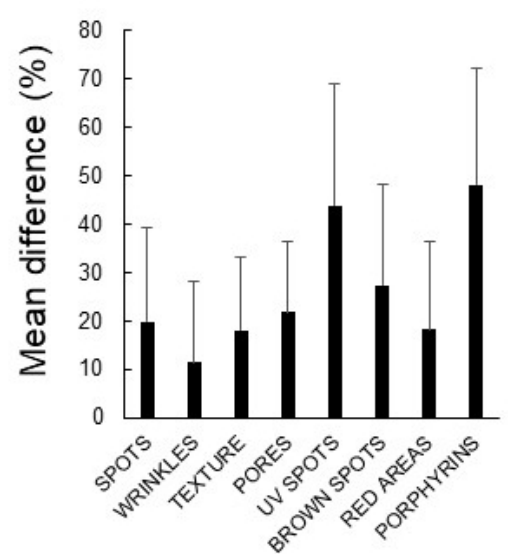

Figure 1. Reproductivity of forearm skin parameters measured by digital imaging system. Eight skin characteristics (SPOTS, WRINKLES, TEXTURE, PORES, UV SPOTS, BROWN SPOTS, RED AREAS, and PORPHYRINS) of forearm skin were analyzed by VISIA in the 38 patients included in this study. Each parameter was separately evaluated on left and right forearms in all subjects to prove its reproducibility, and percentage difference between the two evaluations in each individual was calculated as difference of scores/ larger scores $\times 100$ in each patient. The mean percentage differences of each parameter + standard deviation (SD) are shown on the ordinate.

$3.230 \pm 1.591$, UV SPOTS; $5.761 \pm 3.421$, BROWN SPOTS; $6.704 \pm 1.095$, PORPHYRINS; $0.084 \pm$ 0.0891 ) and SSc forearm- (SPOTS; $2.391 \pm 1.032$, TEXTURE; $4.898 \pm 1.864$, PORES; $2.624 \pm 1.299$, UV SPOTS; $9.835 \pm 11.695$, BROWN SPOTS; 5.798 \pm 5.700 , PORPHYRINS; $0.0616 \pm 0.108)$ compared with those with VEDOSS (SPOTS; $2.362 \pm 1.183$, TEXTURE; $4.738 \pm 1.360$, PORES; $2.234 \pm 1.279$, UV SPOTS; $5.999 \pm 5.138$, BROWN SPOTS; $4.898 \pm$ 3.030, PORPHYRINS; $0.0169 \pm 0.0188)$. By KruskalWallis test, out of the eight parameters there were no significant differences except for PORPHYRINS. On the other hand, for example, although telangiectasia is also a common feature of SSc skin, the scores of RED AREAS were not significantly different among the four groups. Furthermore, contrary to our expectation based on the previous analysis of SSc faces (13), the scores of WRINKLES, TEXTURE, or PORES were not significantly different.

Mann-Whitney tests showed statistical significance in the score of PORPHYRINS between SSc forearmand VEDOSS groups $(p=0.044)$ and between SSc forearm + and VEDOSS groups $(p=0.012)$ (Figure 2). There was no significant difference between the other groups. Increased porphyrins levels may therefore be a specific change to SSc with or without skin sclerosis of the forearm.

3.3. Correlations of PORPHYRINS scores with MRSS or ACR/EULAR 2013 scores in patients with SSc

To confirm the possibility that PORPHYRINS scores can be related to systemic disease activity in patients 
with SSc, we next examined the correlation between PORPHYRINS scores and MRSS or ACR/EULAR2013 scores. However, PORPHYRINS did not show correlation with MRSS $(\mathrm{R}=0.20$, Figure $3 \mathrm{~A})$ and ACR/ EULAR2013 scores $(R=0.047$, Figure 3B). Therefore, although PORPHYRINS scores were increased in SSc forearm skin, we could not prove their direct correlation with systemic disease activity.

\section{Discussion}

VISIA digital imaging system is commonly used for facial analysis in the field of cosmetic dermatology. There have been several studies on facial skin characteristics in patients with hyperpigmented spots and acne using the system (14). We have also performed facial skin analysis of SSc patients using VISIA (13). In that report, we found the severity of WRINKLES, TEXTURE, and PORES were significantly lower in patients with SSc than in control subjects. Among them, WRINKLES showed better correlation with MRSS (8).
However, the usefulness for the early diagnosis could not be evaluated due to the lack of disease controls in the early stage (e.g., VEDOSS).

In the present study, we performed forearm skin analysis using the VISIA system for the first time. The skin condition of fingers and the hand can be affected by ulcers, rings, and bracelets, so we instead focused on the forearm, which is not affected by these factors. The mean percentage differences between two independent measurements of eight parameters were less than 2-fold, indicating the reproducibility of all parameters by our method. Our method can thus be considered as a new option in evaluation of forearm skin characteristics.

Comparison among the four patient groups showed the scores of WRINKLES, TEXTURE, PORES, and PORPHYRINS were higher in SSc forearm+ group and $\mathrm{SSc}$ forearm- group than in control subjects. Also, the scores of SPOTS, TEXTURE, PORES, UV SPOTS, BROWN SPOTS, and PORPHYRINS were elevated in patients in the SSc forearm+ group and SSc forearmgroup compared with those in the VEDOSS group.

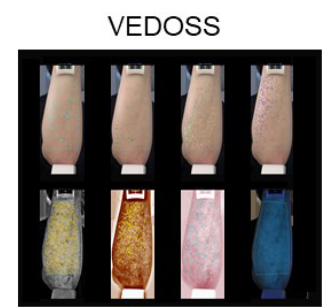

SSc forearm -

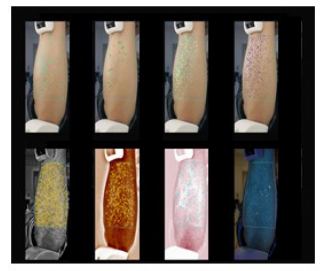

B

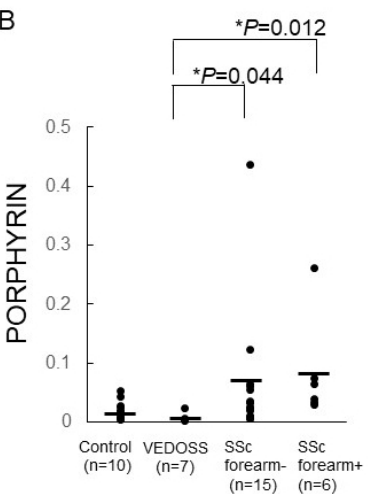

Figure 2. Objective computer assessments of PORPHYRIN levels. (A) A representative photograph showing comparison of a patient with very early diagnosis of systemic sclerosis (VEDOSS) and a SSc patient without skin sclerosis of the forearm (SSc forearm-). Upper row: SPOTS, WRINKLES, TEXTURE, PORES. Lower row: UV SPOTS, BROWN SPOTS, RED AREAS, PORPHIRINS. (B) The score of PORPHIRINS in VEDOSS patients (VEDOSS), SSc patients without skin sclerosis of the forearm (SSc forearm-), SSc patients with skin sclerosis of the forearm (SSc forearm+), and in control subjects (Control) are plotted along the ordinate. $P$-values are determined by Mann-Whitney $U$-test.

A

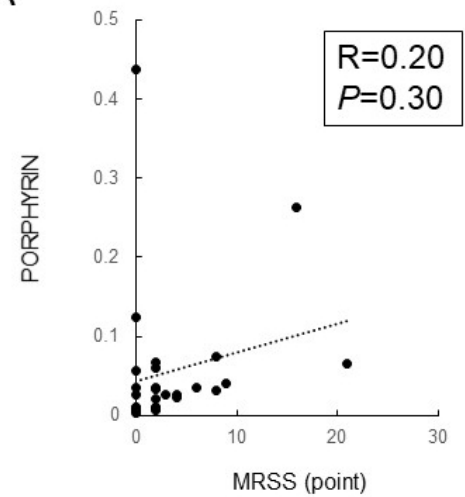

B

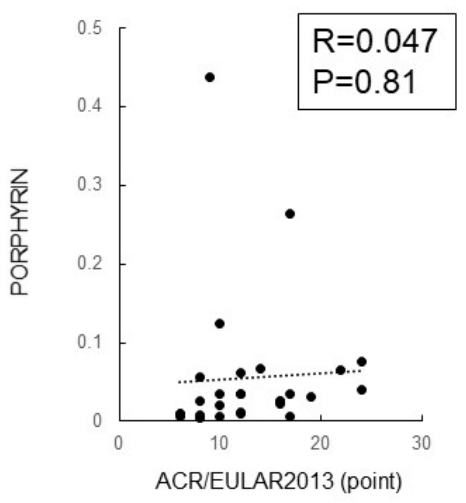

Figure 3. Correlation of PORPHIRINS scores with MRSS or ACR/EULAR2013 score in SSc patients. (A) Correlation of MRSS with PORPHIRINS scores in SSc patients. (B) Correlation of ACR/EULAR2013 score with PORPHIRINS scores in SSc patients. Correlations were assessed by Pearson's correlation coefficient. 
Additionally, unlike in the previous study focusing on SSc facial analysis, out of the eight parameters there was significant difference in this study among the four groups only in PORPHYRINS scores. There was no correlation of PORPHYRINS scores with MRSS or ACR/EULAR scores, so they may not be useful in evaluation of systemic disease activity. PORPHYRINS scores can be an early diagnostic tool, however, because they can be used to differentiate VEDOSS from early or mild SSc cases in whom skin sclerosis is not yet present in the forearm.

Porphyrins are products that play roles in heme metabolism of liver or bone marrow, and the accumulation of porphyrin in the skin is found in several diseases, especially acnes or porphyria cutanea tarda (PCT). In PCT known to cause SSc-like skin sclerosis, pigmentation, blisters, skin fragility, erosion and scar formation, there is a hypothesis that SSclike sclerosis results from phototoxicity of porphyrin (7). Actually, urine levels of porphyrin precursors (i.e., delta-aminolevulinic acid and porphobilinogen) detected by spectrophotometry are reported to be increased in patients with SSc (15). Our results are consistent with these notions. As a possible mechanism of skin sclerosis by porphyrin, uroporphyrin I reportedly induces the production of collagen fibers from normal human cultured fibroblasts (16-18), and the increased levels of porphyrin precursors result in an overgrowth of collagen fibers (15). In addition, the presence of coproporphyrin in the skin produces oxygen radicals (19), which may further cause vasculopathy such as Raynaud's phenomenon and immunodysfuction such as autoantibodies in SSc (20). Moreover, a phase III clinical trial of MT-7117, a selective melanocortin 1 receptor agonist dersimelagon, in patients with erythropoietic protoporphyria and X-linked protoporphyria with a history of photosensitivity began in June 2020. Subsequently, a global phase 2 DECODE study of MT-7117 for the treatment of diffuse cutaneous $\mathrm{SSc}(\mathrm{dcSSc})$ was also initiated in the United States, Canada and in Europe. Study of porphyrins may therefore lead to the development of novel therapies as well as early diagnosis.

On the other hand, there are some limitations in the present study. First, the data of healthy subjects was not available, because they rarely visit our hospital as a center in the area. Thus, we could not compare the VISIA data of SSc patients with those of healthy controls. Next, we have not directly measured porphyrin levels in skin, blood or urine. Therefore, the actual increase of porphyrins in the patients is not confirmed. This is a pilot study, and the number of patients included is rather low to allow reliable conclusion. To confirm the result, further detailed researches with larger number of samples and confirmation with other experimental methods are necessary in the future.

In summary, the scores of WRINKLES, TEXTURE,
PORES, and PORPHYRINS were higher in SSc forearm+ and SSc forearm- groups than in control subjects. Scores of SPOTS, TEXTURE, PORES, UV SPOTS, BROWN SPOTS, and PORPHYRINS were elevated in patients in SSc forearm+ and SSc forearm- groups compared with those in VEDOSS group. We found statistical significance in the score of PORPHYRINS between SSc forearm- and VEDOSS, and between SSc forearm+ and VEDOSS. Therefore, they can be used to differentiate VEDOSS from early or mild SSc cases, which is sometimes clinically problematic. Furthermore, it may have great potential as a new therapeutic tool. Our study also suggests that the porphyrin research will lead to a better understanding of SSc pathogenesis.

\section{Acknowledgements}

We acknowledge proofreading and editing by Benjamin Phillis at the Clinical Study Support Center, Wakayama Medical University.

\section{Funding: None.}

Conflict of Interest: The authors have no conflicts of interest to disclose.

\section{References}

1. Makino K, Jinnin M, Makino T, Kajihara I, Fukushima $\mathrm{S}$, Inoue Y, Ihn H. Serum levels of soluble carbonic anhydrase IX are decreased in patients with diffuse cutaneous systemic sclerosis compared to those with limited cutaneous systemic sclerosis. Biosci Trends. 2014; 8:144-148.

2. Kawashita Y, Jinnin M, Makino T, Kajihara I, Makino K, Honda N, Masuguchi S, Fukushima S, Inoue Y, Ihn H. Circulating miR-29a levels in patients with scleroderma spectrum disorder. J Dermatol Sci. 2011; 61:67-69.

3. Tabata K, Jinnin M, Furukawa K, Tani S, Okuhira H, Mikita N, Fujii T. Finger sweating levels evaluated by video capillaroscopy system are increased in patients with systemic sclerosis compared to preclinical stage patients. Drug Discov Ther. 2021; 14:325-329.

4. van den Hoogen F, Khanna D, Fransen J, et al. 2013 classification criteria for systemic sclerosis: an American college of rheumatology/European league against rheumatism collaborative initiative. Ann Rheum Dis. 2013; 72:1747-1755.

5. Matucci-Cerinic M, Allanore Y, Czirják L, et al. The challenge of early systemic sclerosis for the EULAR Scleroderma Trial and Research group (EUSTAR) community. It is time to cut the Gordian knot and develop a prevention or rescue strategy. Ann Rheum Dis. 2009; 68:1377-1380.

6. Clements PJ, Lachenbruch PA, Seibold JR, et al. Skin thickness score in systemic sclerosis: an assessment of interobserver variability in 3 independent studies. J Rheumatol. 1993; 20:1892-1896.

7. Linming F, Wei H, Anqi L, Yuanyu C, Heng X, Sushmita P, Yiming L, Li L. Comparison of two skin imaging 
analysis instruments: The VISIA ${ }^{\circledR}$ from Canfield $v s$. the ANTERA 3D ${ }^{\circledR}$ CS from Miravex. Skin Res Technol. 2018; 24:3-8.

8. Goldsberry A, Hanke CW, Hanke KE. VISIA system: a possible tool in the cosmetic practice. Drugs Dermatol. 2014; 13:1312-1314.

9. Kislevitz M, Lu KB, Wamsley C, Hoopman J, Kenkel J, Akgul Y. Novel use of non-invasive devices and microbiopsies to assess facial skin rejuvenation following laser treatment. Lasers Surg Med. 2020; 52:822-830.

10. Kuo SCH, Huang F, Chi SY, Lin HP, Chien PC, Hsieh $\mathrm{CH}$. Investigate the improvement of facial skin texture with the VISIA system after total thyroidectomy. BMC Surg. 2021; 21:94.

11. Ichibori R, Fujiwara T, Tanigawa T, Kanazawa S, Shingaki K, Torii K, Tomita K, Yano K; Osaka Twin Research Group, Sakai Y, Hosokawa K. Objective assessment of facial skin aging and the associated environmental factors in Japanese monozygotic twins. J Cosmet Dermatol. 2014; 13:158-163.

12. Lee SJ, Seok J, Jeong SY, Park KY, Li K, Seo SJ. Facial pores: Definition, causes, and treatment options. Dermatol Surg. 2016; 42:277-285.

13. Sawamura S, Jinnin M, Kajihara I, Makino K, Aoi J, Ichihara A, Makino T, Fukushima S, Ihn H. Do scleroderma patients look young?: Evaluation by using facial imaging system. Drug Discov Ther. 2017; 11:342345.

14. Takahashi Y, Fukushima Y, Kondo K, Ichihashi M. Facial skin photo-aging and development of hyperpigmented spots from children to middle-aged Japanese woman. Skin Res Technol. 2017; 23:613-618.

15. Osiecka B J, Ziółkowski P, Gamian E, Nockowski P. The evaluation of porphyrin content in patients with systemic scleroderma: Preliminary study. Dermatologia Kliniczna. 2006; 8:249-251.

16. Grossman ME, Bickers DR, Poh-Fitzpatrick MB, Deleo VA, Harber LC. Porphyria cutanea tarda. Clinical features and laboratory findings in 40 patients. Am J Med. 1979; 67:277-286.

17. Epstein JH, Tuffanelli DL, Epstein WL. Cutaneous changes in the porphyrias. A microscopic study. Arch Dermatol. 1973; 107:689-698.

18. Varigos G, Schiltz JR, Bickers DR. Uroporphyrin I stimulation of collagen biosynthesis in human skin fibroblasts. A unique dark effect of porphyrin. J Clin Invest. 1982; 69:129-135.

19. Ryu A, Arakane K, Koide C, Arai H, Nagano T. Squalene as a target molecule in skin hyperpigmentation caused by singlet oxygen. Biol Pharm Bull. 2009; 32:1504-1509.

20. Herrick AL, Rieley F, Schofield D, Hollis S, Braganza JM, Jayson MI. Micronutrient antioxidant status in patients with primary Raynaud's phenomenon and systemic sclerosis. J Rheumatol. 1994; 21:1477-1483.

Received December 20, 2021; Revised January 31, 2022; Accepted February 4, 2022.

*Address correspondence to:

Chikako Kaminaka, Department of Dermatology, Wakayama Medical University Graduate School of Medicine, 811-1 Kimiidera, Wakayama 641-0012, Japan.

E-mail:kamikami@wakayama-med.ac.jp

Released online in J-STAGE as advance publication February 10, 2022. 